\title{
MICROSIMULATION-BASED PASSENGER CAR EQUIVALENTS FOR HEAVY VEHICLES DRIVING TURBO-ROUNDABOUTS
}

\author{
Orazio Giuffrè ${ }^{1}$, Anna Granà ${ }^{2}$, Sergio Marino ${ }^{3}$, Fabio Galatioto ${ }^{4}$ \\ ${ }^{1,2,3}$ Dept of Civil, Environmental, Aerospace and Materials Engineering; University of Palermo, Italy \\ ${ }^{4}$ Transport Systems Catapult, Milton Keynes, United Kingdom
}

Submitted 30 April 2015; resubmitted 23 September 2015, 2 February 2016; accepted 5 March 2016

\begin{abstract}
Due to its geometric design, turbo-roundabouts impose greatest constraints to the vehicular trajectories; by consequence, one can expect a more unfavourable impact of heavy vehicles on the traffic conditions than on other types of roundabouts. The present paper addresses the question of how to estimate Passenger Car Equivalents (PCEs) for heavy vehicles driving turbo-roundabouts. The microsimulation approach used revealed as a useful tool for evaluating the variation of quality of traffic in presence of mixed fleets (different percentages of heavy vehicles). Based on the output of multiple runs of several scenarios simulation, capacity functions for each entry lane of the turbo-roundabout were developed and variability of the PCEs for heavy vehicles were calculated by comparing results for a fleet of passenger cars only with those of the mixed fleet scenarios. Results show a dependence of PCEs for heavy vehicles on operational conditions, which characterise the turbo-roundabout. Assuming the values of PCEs for roundabouts provided by the 2010 Highway Capacity Manual (HCM), depending on entering manoeuvring underestimation and overestimation of the effect of heavy vehicles on the quality of traffic conditions have been found.
\end{abstract}

Keywords: roundabouts; turbo-roundabouts; passenger car equivalent; heavy vehicles; microsimulation; Aimsun.

\section{Introduction}

Turbo-roundabouts represent a recent scheme of roundabouts, which provide a spiralling traffic flow and require drivers to choose their direction before entering the intersection, since physical barriers mark the lanes on the ring (Fortuijn 2009). As a result of the turboroundabout design, patterns of conflict at entries with one and two conflicting traffic streams can coexist (Fortuijn 2009; Mauro et al. 2015; Tollazzi 2015).

This paper addresses the question of how to estimate Passenger Car Equivalents (PCEs) for heavy vehicles driving turbo-roundabouts. This study starts from the belief that constraints to the vehicular trajectories imposed by the turbo-roundabout design imply a more unfavourable impact of heavy vehicles on the quality of traffic flow than on other roundabouts; thus higher PCEs than the established values (Highway Capacity Manual 2010) are expected. As Huber (1982) proposed, PCEs were calculated by comparing entry-lane capacities both for a fleet of passenger cars only and for a mixed fleet. Estimations of capacity for each entry lane of the turbo-roundabout were obtained by using microsimulation, varying the percentage of heavy vehicles in traffic demand. Microsimulation revealed as a useful tool to evaluate the variation of the traffic quality at turbo-roundabouts in presence of mixed fleets, each having different percentages of heavy vehicles; indeed, a microscopic traffic simulation model has been used to isolate traffic conditions difficult to observe on field, to replicate them to have a significant amount of data. Using Aimsun software it was possible to account for the wide range of traffic conditions on the basic turboroundabout selected as case study. It should be noted that Aimsun software has been applied to turbo-roundabouts only recently; therefore, there are few studies examining turbo-roundabout performances, in particular, when these roundabouts are applied in urban networks or in corridors (Bastos Silva et al. 2015). However, some researchers have used Aimsun for traditional roundabouts in the recent past (Gingrich, Dion 2012; Zhang, Excell 2013; Zenina, Merkuryev 2009).

Nonlinear regression analysis of simulation data allowed to recalculate critical and follow-up headways for mixed fleets, up to $100 \%$ heavy vehicles. Capacity functions were then used to determine how the PCE varies with the percentage of heavy vehicles and circulating 
flows. A larger PCE effect would be expected when the traffic stream contains a significant number of heavy vehicles; this effect should be accounted for in calculating capacity and level-of-services.

\section{Overview of Past Research on PCEs}

Heterogeneity in the vehicular traffic mix, which composes a traffic stream, represents one of the important issues affecting the accuracy of traffic analyses. The characteristics of the heavy vehicles (mainly trucks, recreational vehicles and large buses) vary from those of passengers cars since heavy vehicles are larger in dimensions and often inferior to passenger cars in performances. However, despite heavy vehicles can usually represent less than $30 \%$ of total vehicles of a traffic mix, heavy vehicles have an important impact on the traffic stream. The effect of heavy vehicles on traffic flow has been accounted for through the use of PCEs; indeed, PCEs approximate the effect of heavy vehicles and are expressed as multiples of the effect of an average passenger car (Al-Kaisy 2006; Praveen, Arasan 2013). Many studies have shown the factors on which PCEs can depend: dimensions, power, speed, acceleration and braking characteristics of the vehicle, road geometric characteristics including gradients, curves, access controls, type of road (rural or urban), presence and type of intersection. PCEs are therefore considered essential in carrying out most traffic analyses since incorporating the impact of heavy vehicles on traffic operations makes the performance analysis of roads or intersections more accurate.

The impact of heavy vehicles on traffic operations has been an interesting topic since the first editions of the Highway Capacity Manual (Highway Capacity Manual... 1950, 1965, 1994). However, the PCEs used in the HCM procedures account for the effect of dimensions and performance of heavy vehicles only under steadystate conditions; the inferior acceleration performances exhibited after the onset of congestion are not incorporated (Al-Kaisy et al. 2005). However, HCM PCEs have been used to conduct analyses for all traffic conditions (from free-flow to congested-flow conditions). Because capacity is often realized at saturated operations, the use of HCM PCEs for demand/capacity analysis during queuing operations is expected to estimate improperly the effect of heavy vehicles. The acceleration and deceleration cycles, as normally experienced during congestion or stop-and-go conditions, impose an extra limitation on the performance of heavy vehicles.

Al-Kaisy (2006) introduced practical insights into the limitations and appropriate use of the current HCM PCEs. The recent versions of the Highway Capacity Manual $(2000,2010)$, indeed, provide a simplified approach to quantifying the effect of heavy vehicles on the traffic stream; PCEs represent the full spectrum of heavy vehicles in the traffic mix regardless of performance and the full range of traffic conditions regardless of levelof-service. In the HCM approach, PCE for roundabouts is not sensitive to the performance of heavy vehicles or traffic level, but it is independently from the vehicle type and the percentage of heavy vehicles.
It is worth mentioning that several methodologies have been used to calculate PCEs for highways and intersections. Definitions of equivalency based on the heavy vehicle effect on different parameters have been proposed. To name a few, methods for PCE estimations can be based on flow rates and density (Huber 1982; Webster, Elefteriadou 1999), headways (Anwaar et al. 2011), queue discharge flow (Al-Kaisy et al. 2002), travel time (Keller, Saklas 1984), delay (Cunagin, Messer 1983) and so on. One can remember Craus et al. (1980), which considered the traffic delays caused by heavy vehicles and opposing traffic; they obtained and proposed a new set of PCEs which exhibited a similar fluctuation of the HCM PCEs, but were characterised by significantly lower values for slow heavy vehicle speeds; these results reflected more closely the actual disturbance and delay caused by trucks as well as consideration of traffic in the opposite lane.

Significant differences were found among PCEs from different methods especially in heterogeneous traffic environment (Adnan 2014). Most studies and researches used traffic simulation to obtain equivalent flows for a wide combination of flows and geometric conditions. Elefteriadou et al. (1997) found values of PCEs for freeways, two-lane highways, and arterials by using simulation. They indicated that some variables, such as percentage of trucks, did not always have the expected effect on PCEs; in turn, other variables, such as vehicle type, can result crucial in calculations. However, Elefteriadou et al. (1997) found that major differences in PCEs occurred on longer and steeper grades, whereas great variability in PCEs as a function of the weight/horsepower ratio and vehicle length was also shown. Webster and Elefteriadou (1999) included in their study a wide range of traffic conditions and derived PCEs based on density by using simulation technique. The following inferences were made through their study: PCE value tends to increase with traffic flow, free-flowspeed, and grade/length of grade, whereas PCE value tends to decrease with an increase in heavy vehicle percentage and number of lanes. Demarchi and Setti (2003) studied the limitation of PCE derivation with more than one truck type. Geistefeldt (2009) estimated PCEs based on capacity variability and simulated different factors affecting PCEs. Based on the concept of stochastic capacities, he estimated the capacity distribution functions; with a determination of the equivalency factor for which the coefficient of variation of the capacity distribution function was minimal, PCEs for heavy vehicles were derived. However, the calculation of PCEs for freeways and highways seems us off the scope of this paper; thus, we recommend the reader more interested to refer to the wide literature available on PCEs for these road infrastructures.

It should be also said that only a few studies were based on field data or have calibrated PCEs for a specific road infrastructure. A large community of academics and practitioners during the last decade have been using Aimsun in a large array of applications. However, techni- 
cal literature still presents few studies related to the calculation of PCEs for heavy vehicles at roundabouts (e.g. Jia et al. 2008; Macioszek 2012; Lee 2014). The Highway Capacity Manual $(2000,2010)$ proposes PCEs for heavy vehicles equal to two for all types of roundabouts for every traffic condition; nothing is proposed for the turbo-roundabouts. Thus, there is a gap in the current literature of assessment of PCEs specifically for turboroundabouts that this paper aims to address.

\section{Calibration in Simulation Modelling}

According to Barceló (2010), calibration of a traffic microsimulation model is an iterative process by which some model parameters are changed and adjusted, and model outputs are compared with a set of empirical data until a predefined level of agreement between the two data sets is achieved. In the context of microsimulationbased modelling, calibration was held by ensuring that Aimsun software for each entry lane of the turbo-roundabout gave simulated capacities close to those which could be derived from the Hagring (1998) model. According to Vasconcelos et al. (2012), not only this model generated accurate estimates for the complete range of circulating flows, but it also satisfactorily described the effect of traffic distribution in the circulating lanes.

The gap-acceptance parameters on field collected by Fortuijn (2009) were introduced into the adopted capacity formulation. Fortuijn (2009), indeed, estimated critical and follow up headways for basic turbo-roundabouts operating in the Netherlands. The values of the critical and follow-up headways were differentiated by leg (major or minor entries) and by lane (right or left entry lane). Only for the left entry lane at minor entries, Fortuijn (2009) measured two values of the critical headway: $T_{c i}$ for the inner circulating lane and $T_{c e}$ for the outer circulating lane. Starting from data reported by Fortuijn (2009), in the present paper we assumed gapacceptance parameters as shown in Table 1. Since the values of critical headway were measured for different sites, each of them with a proper sample size, a weight- ed mean of these parameters was used. By this way, the abstraction in theoretical modelling was tempered by the realism of behavioural parameters (Vasconcelos et al. 2013, 2014). Capacities derived from the Hagring (1998) model for each entry lane (Table 1), were used as reference for calibration purposes. They play the role of the empirical data usually employed in the calibration process. Table 1 also shows entry-lane capacity formulas derived from the Hagring (1998) model: left-lane capacity from minor entries were calculated by Eq. (1) as a function of the inner $Q_{c i}$ and the outer $Q_{c e}$ circulating flows, whereas right- and left-lane capacity from major entries and right-lane capacity from minor entries were calculated by Eq. (2) as a function of the whole circulating traffic flow $Q_{c}$; a minimum arrival headway $\Delta$ of $2.10 \mathrm{~s}$ was selected (Giuffrè et al. 2012).

Fig. 1 shows the the sketch of the basic turboroundabout selected in the application of the methodology for the calculation of PCEs; on the right the network turbo-roundabout model in Aimsun is also shown. It should be noted that a priority was also created for the legs of the turbo-roundabout: legs 2 and 4 were considered as major entries, whereas legs 1 and 3 were considered as minor entries.

With reference to the turbo-roundabout in Fig. 1, the following radii for roadway edges were selected: with reference to the inside roadway, an inner radius $R_{1}$ of 12 $\mathrm{m}$ and an outer radius $R_{2}$ of $17 \mathrm{~m}$; with reference to the outside roadway, an inner radius $R_{3}$ of $17.30 \mathrm{~m}$ and an outer radius $R_{4}$ of $22.15 \mathrm{~m}$. The cross section elements included an inside lane width of $4.50 \mathrm{~m}$, an outside lane width of $4.20 \mathrm{~m}$, whereas edge strip, and median strip widths were selected based on the Dutch and Slovenian manuals (e.g. CROW 2008; Tollazzi 2015).

For calibration purposes, the entry-lane capacities derived from Eqs (1-2) were compared with simulation outputs. In order to calculate the capacity values at each entry lane, saturation conditions were simulated on an entry lane of the turbo-roundabout time by time considered. For this calculation we proceed assigning $\mathrm{O} / \mathrm{D}$ matrices such as reproducing the circulating flows, namely

Table 1. Gap acceptance parameters and capacity formulas at turbo-roundabout entries

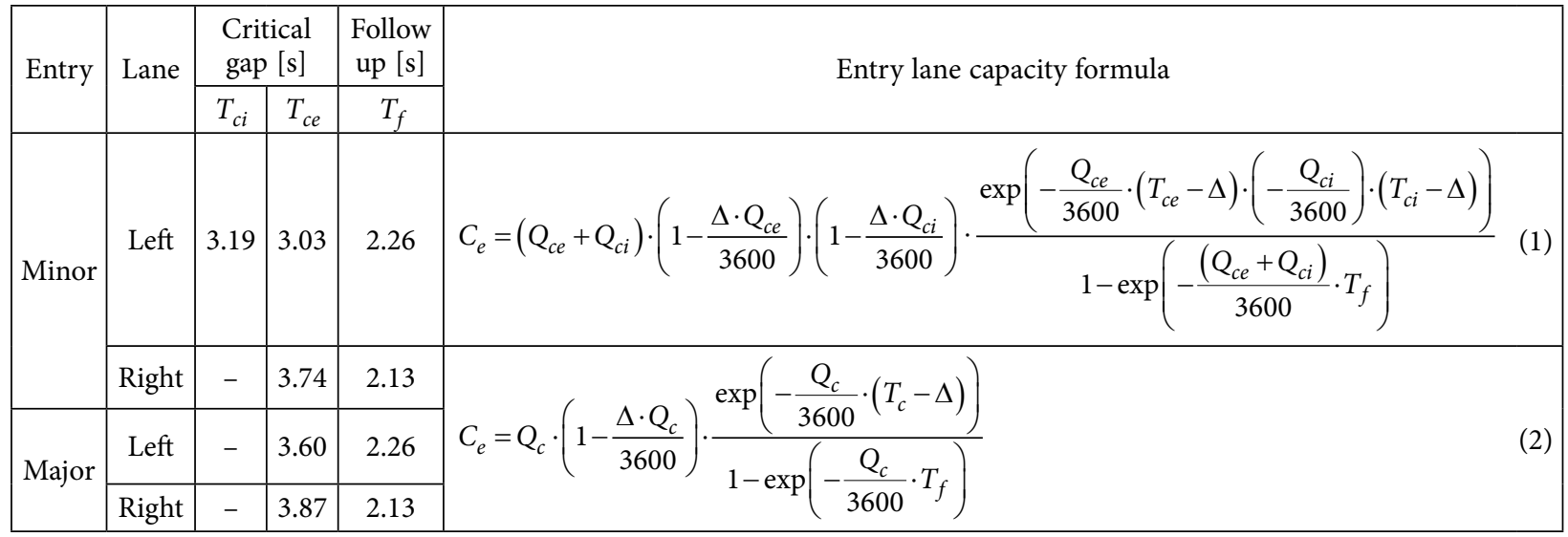

Note: that $T_{c i}$ is the critical gap for the inner lane of the entry and $T_{c e}$ is the critical gap for the outer lane at entry. 
the (antagonist) circulating flow $Q_{c}$, from $0 \mathrm{veh} / \mathrm{h}$ to $1700 \mathrm{veh} / \mathrm{h}$ with a step of $100 \mathrm{veh} / \mathrm{h}$. For the left-entry lane only two circulating flows - one in the inner $Q_{c i}$ and one in the outer $Q_{c e}$ circulating lane - were reproduced. $\mathrm{O} / \mathrm{D}$ matrices guaranteed saturation conditions at entries, so that the number of vehicles entering the turbo-roundabout represented the capacity for a specific entry lane.

In order to have capacity predictions similar to capacity estimates derived from Hagring (1998), it was decided to calibrate the values of that parameters with particular influence in the gap-acceptance process. After performing several iterations, manually adjusting different combinations of values of some default parameters
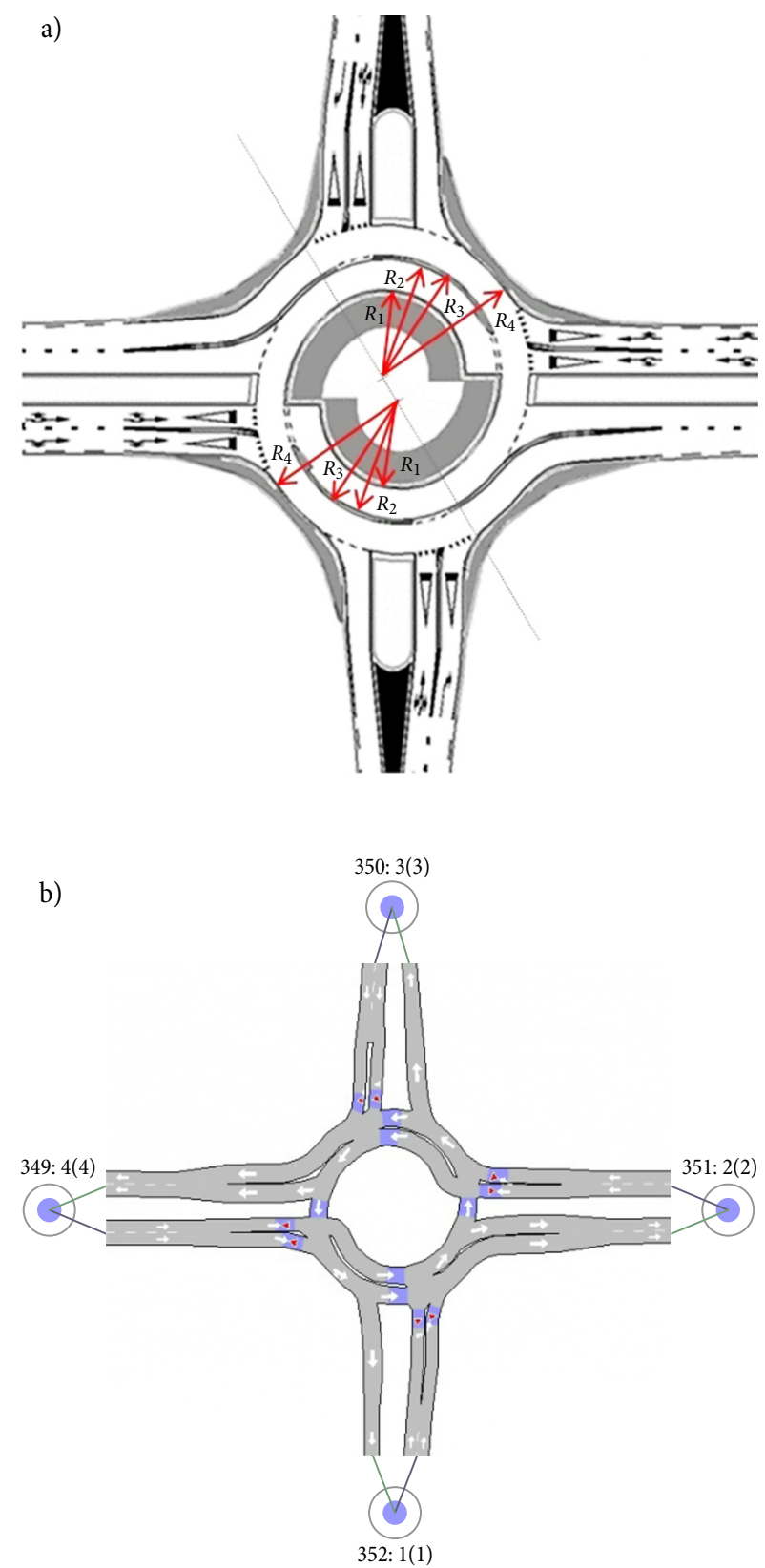

Fig. 1. The sketch of the basic turbo-roundabout (a) and the turbo-roundabout model in Aimsun (b) in Aimsun 6.1, a minimum headway (the time in seconds between the leader and the follower vehicle) of $1.70 \mathrm{~s}$ was used instead of the default value of $0.00 \mathrm{~s}$, whereas a reaction time (the time in seconds it takes a driver to react to speed changes in the preceding vehicle) of $1.00 \mathrm{~s}$ was used instead of the default value of $1.35 \mathrm{~s}$.

According to Barceló (2010) a method that has been widely used in validating traffic micro-simulation models, in particular when only aggregated values are available (i.e. flow counts at detection stations aggregated to the hour, capacity values, etc.), has been to analyse the scattergram or, preferably as an alternative, to use a global indicator as the GEH index. Thus, the scattergram analysis was developed to compare the observed and the simulated capacities at each entry lane of the examined turbo-roundabout. The regression lines of observed versus simulated capacities for each entry lane were plotted along with the 95\% Prediction Interval (95\% PI); see e.g. Mauro et al. (2014) for other applications. Fig. 2 shows an example of the capacity curve versus simulation outputs, whereas Figs 3 and 4 depict the regression lines of observed versus simulated capacities for each entry lane and 95\% PI. One can observe that the bandwidth varied for the different entry lanes, given the greater or lower dispersion observed from case to case; however, the $R^{2}$ values and the fact that most of points fell almost entirely within the confidence band of the regression lines led to the conclusion which the model could be accepted as significantly close to the reality. In order to show how the residuals change by using calibrated models and real measurements among the entry lanes, Table 2 reports the root mean squared normalized errors, which provides information on the magnitude of the errors relative to the average measurement; the mean absolute percent error was also calculated as supplemental parameter to measure the size of the error in percentage terms. Despite the model would have been accepted, the GEH index was also used as criterion for acceptance, or otherwise rejection, of the model (Barceló 2010). Since the deviation of each simulated value with respect to the measurement for each entry lane was smaller than 5 in $100 \%$ of the cases, the model was accepted as significantly able to reproduce local conditions and traffic behaviour.

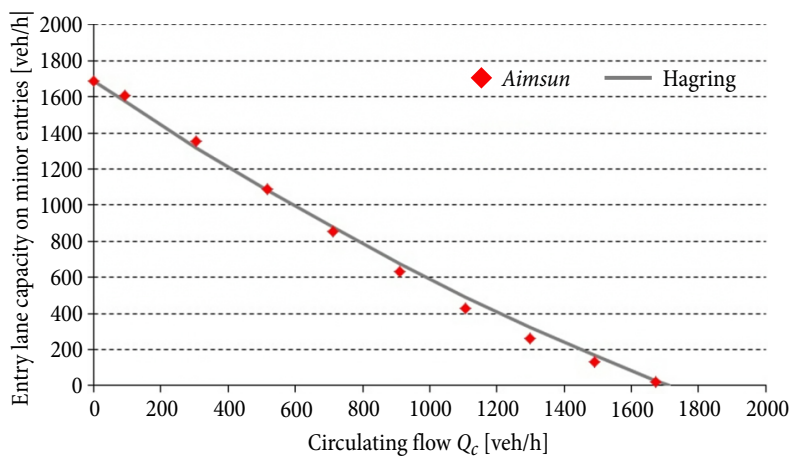

Fig. 2. Right lane capacity on minor entries: simulation vs Hagring data 
Table 2. Variations of the percentage error by entry lane

\begin{tabular}{|c|c|c|c|}
\hline \multirow{2}{*}{ Entry } & Lane & $R M S N E=\sqrt{\frac{1}{N} \sum_{i=1}^{N}\left(\frac{x_{i}-y_{i}}{y_{i}}\right)^{2}}$ & MAPE $=\frac{100}{N} \sum_{i=1}^{N}\left|\frac{x_{i}-y_{i}}{y_{i}}\right|[\%]$ \\
\hline \multirow{2}{*}{ Minor } & Right & 0.145 & 10.08 \\
\cline { 2 - 4 } & Left & 0.183 & 12.30 \\
\hline \multirow{2}{*}{ Major } & Right & 0.143 & 8.09 \\
\cline { 2 - 4 } & Left & 0.025 & 1.93 \\
\hline
\end{tabular}

Note: that $x_{i}$ and $y_{i}$ are the $i$-th measured and observed value and $N$ are the measured/observed points.

a)
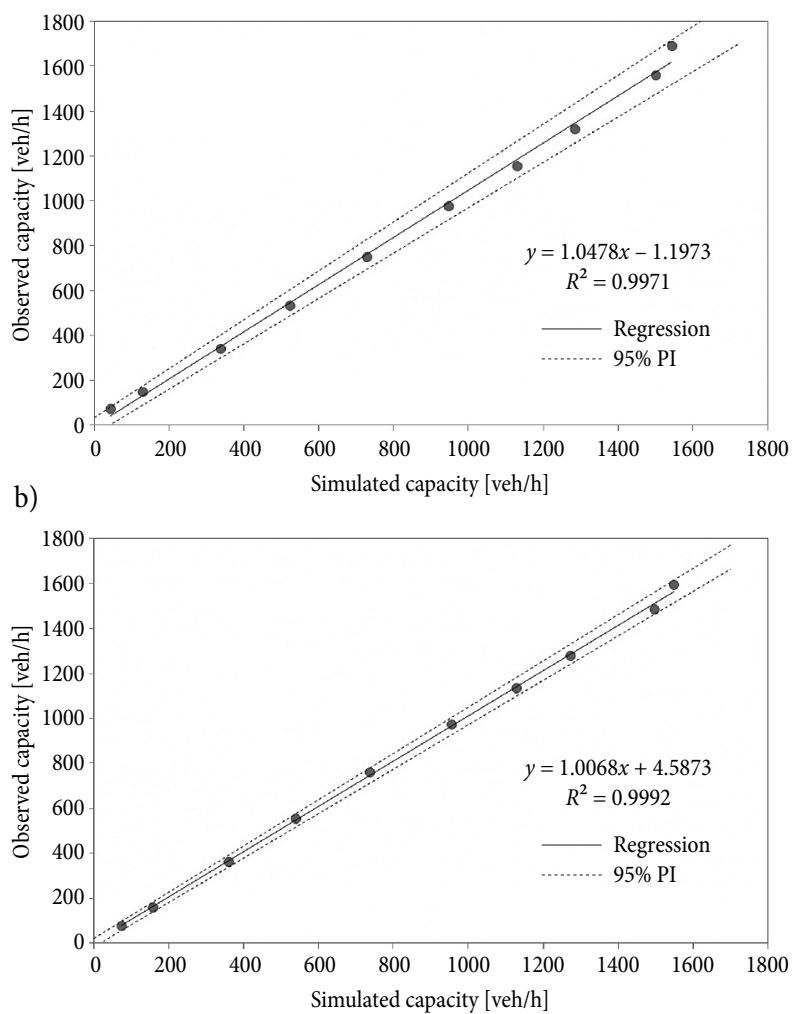

Fig. 3. Observed versus simulated capacities at major entries: $\mathrm{a}$ - the right-lane; $\mathrm{b}$ - the left-lane

\section{Calculating PCEs at Turbo-Roundabouts}

This section addresses the question of how to develop PCEs for heavy vehicles driving turbo-roundabouts. PCEs were estimated as a function of different percentages of heavy vehicles in the traffic demand and circulating flows of passenger cars only. The heavy vehicles mix included trucks having Aimsun default values for their attributes (maximum length of $10 \mathrm{~m}$; maximum desired speed of $85 \mathrm{~km} / \mathrm{h}$ with a range $70-100 \mathrm{~km} / \mathrm{h}$; maximum acceleration of $1 \mathrm{~m} / \mathrm{s}^{2}$ with a range $0.6-1.8 \mathrm{~m} / \mathrm{s}^{2}$; maximum deceleration of $5 \mathrm{~m} / \mathrm{s}^{2}$ with a range $4-6 \mathrm{~m} / \mathrm{s}^{2}$ ).

\subsection{Method of PCE Estimation}

Assuming a circulating flow being made of passenger cars only as reference case, the effect of a single class of a)

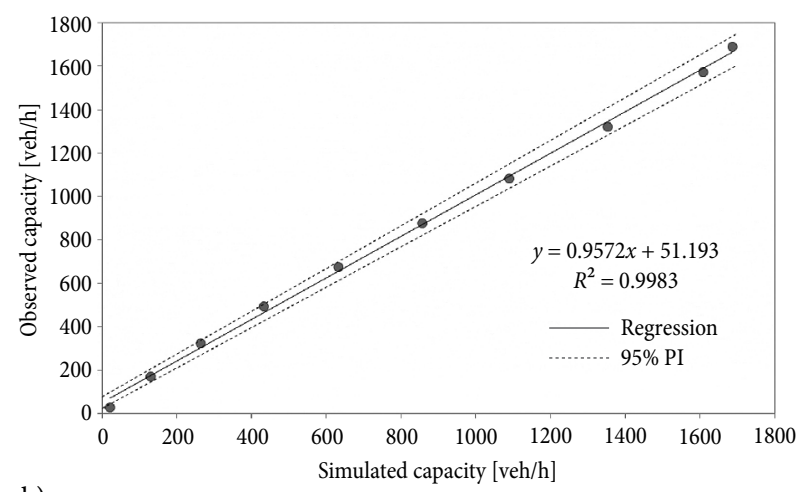

b)

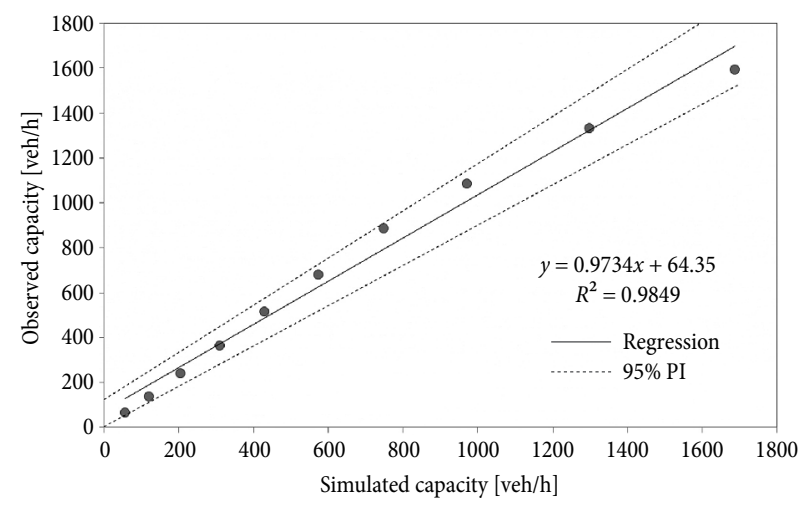

Fig. 4. Observed versus simulated capacities at minor entries: $\mathrm{a}$ - the right-lane; $\mathrm{b}$ - the left-lane

heavy vehicles on turbo-roundabout operations has been determined; for each entry lane the PCE is estimated by comparing the capacity $C_{c a r}$ that would occur in presence of a traffic demand of passenger cars only and the capacity $C_{p}$ corresponding to a traffic demand characterised by a $p$ percentage of heavy vehicles. Based on Huber's (1982) study we adopted the following equation: $C_{c a r}=(1-p) \cdot C_{p}+p \cdot C_{p} \cdot E_{t}$, in which $C_{p}$ is the entering heterogeneous flow in saturation conditions including both the share of passenger cars $(1-p) C_{p}$ and the share of heavy vehicles $\left(p \cdot C_{p}\right)$, multiplied by the equivalent factor $E_{t}$ for reasons of homogeneity. Thus, the equivalent factor $E_{t}$ is given by:

$$
E_{t}=\frac{C_{c a r}-(1-p) \cdot C_{p}}{p \cdot C_{p}} .
$$


Since the passenger car is the base vehicle, the capacity function $C_{c a r}$ is called the base curve, whereas $C_{p}$ is the capacity function for a mixed fleet. Depending the $C_{c a r}$ and $C_{p}$ functions on the circulating flow, $E_{t}$ is depending on the whole circulating flow $Q_{c}$ for left- and right-lane on major entries and right-lane on minor entries; $E_{t}$ is depending on two circulating flows $-Q_{c e}$ and $Q_{c i}$ - for left-lanes on minor entries. In order to develop the capacity functions $C_{c a r}$ and $C_{p}, \mathrm{O} / \mathrm{D}$ matrices were assigned in Aimsun as above introduced. The traffic demand was each time composed of different mixed fleets (100\% passenger cars, $10 \%, 20 \%$, up to $100 \%$ heavy vehicles). For the right- and left-lane on major entries and right-lane on minor entries, 10 replications were used for each mixed fleet at different values of circulating flow. For the left-lanes on minor entries, 10 replications were used for each mixed fleet and for 7 combinations of circulating flows $\left(Q_{c i}=0, Q_{c e}=\operatorname{var} ; Q_{c i} / Q_{c e}=0.33 ; Q_{c i} l\right.$ $Q_{c e}=0.5 ; Q_{c i} / Q_{c e}=1 ; Q_{c i} / Q_{c e}=2 ; Q_{c i} / Q_{c e}=3 ; Q_{c i}=$ var, $\left.Q_{c e}=0\right)$; these combinations approximate a gradual variations of the traffic variation on the two circulating lanes. Simulation data were then used to obtain the $C_{c a r}$ and $C_{p}$ functions for each entry lane of the turboroundabout and for the different mixed fleets (different percentages of heavy vehicles).

\subsection{Regression Analysis}

Starting from the above assumptions, the Hagring (1998) model (Eqs (1-2)) was adopted as the functional form best suited to perform regression on simulation data.
The critical and the follow-up headways were regarded as the parameters to be estimated by regression; Mathematica 9.0 software was used. The results of regressions for the right-lane and left-lane on minor entries are shown in Tables 3 and 4.

Results of statistical regressions showed that the increase in the percentages of heavy vehicles corresponded to an increase in the parameter estimations at all the entry; in turn, the increase in the percentages of heavy vehicles corresponded to a reduction in the simulated values of entry capacity, as the chance of insertion of heavy vehicles into headways in major streams are gradually being reduced due to poorer kinematic performances of heavy vehicles than passenger cars. The values estimated by statistical regression should be considered as values of the behavioural parameters related to heterogeneous user populations, characterised by specific percentages of heavy vehicles. Moreover, having considered populations made by heavy vehicles only allowed us to determine the values of the parameters that explain the behaviour implemented by this single user category, for each entering manoeuvre at turbo-roundabout.

The capacity functions were then developed by each entry lane of the turbo-roundabout and for different mixed fleets options. In all cases, the increase in the circulating flow corresponded to a reduction in the capacity values, especially when higher percentages of heavy vehicles characterised the traffic demand. Fig. 5 shows the simulated points and the capacity functions for the left-lane on major entries (Fig. 5a) and for the right-lane on major entries (Fig. 5b).

Table 3. Results of regressions for the right-lane on minor entries

\begin{tabular}{|c|c|c|c|c|c|c|}
\hline Fleet & Parameter & Est (s.e.) $[\mathrm{s}]$ & $R^{2}$ & $t$-value & $p$-value & confidence interval $[\mathrm{s}](\alpha=0.05)$ \\
\hline \multirow{2}{*}{$10 \% \mathrm{hv}$} & $T_{c}$ & $4.54(0.0652)$ & \multirow{2}{*}{0.99} & 69.67 & $2.01 \cdot 10^{-12}$ & $4.3904-4.6910$ \\
\cline { 2 - 3 } & $T_{f}$ & $2.14(0.0157)$ & & 136.18 & $9.45 \cdot 10^{-15}$ & $2.1046-2.1770$ \\
\hline \multirow{2}{*}{$20 \% \mathrm{hv}$} & $T_{c}$ & $4.92(0.0344)$ & \multirow{2}{*}{0.99} & 142.83 & $6.46 \cdot 10^{-15}$ & $4.8478-4.9945$ \\
\cline { 2 - 3 } \cline { 5 - 6 } & $T_{f}$ & $2.20(0.0081)$ & & 272.35 & $3.69 \cdot 10^{-17}$ & $2.1837-2.2210$ \\
\hline \multirow{2}{*}{$100 \% \mathrm{pc}$} & $T_{c}$ & $4.03(0.1009)$ & \multirow{2}{*}{0.99} & 39.91 & $1.71 \cdot 10^{-10}$ & $3.7931-4.2584$ \\
\cline { 2 - 3 } \cline { 5 - 6 } & $T_{f}$ & $2.08(0.0256)$ & & 81.33 & $5.82 \cdot 10^{-13}$ & $2.0226-2.1407$ \\
\hline
\end{tabular}

Note: hv - stands for heavy vehicles; pc - stands for passenger cars; $\alpha$ - significance level.

Table 4. Results of regressions for the left-lane on minor entries

\begin{tabular}{|c|c|c|c|c|c|c|}
\hline Fleet & Parameter & Est (s.e.) $[\mathrm{s}]$ & $R^{2}$ & $t$-value & $p$-value & confidence interval $[\mathrm{s}](\alpha=0.05)$ \\
\hline \multirow{3}{*}{$10 \% \mathrm{hv}$} & $T_{c i}$ & $3.97(0.0648)$ & \multirow{4}{*}{0.99} & 61.31 & $3.05 \cdot 10^{-59}$ & $3.8421-4.1008$ \\
\cline { 2 - 3 } \cline { 5 - 6 } & $T_{c e}$ & $4.23(0.0667)$ & 63.49 & $3.27 \cdot 10^{-60}$ & $4.1016-4.3680$ \\
\cline { 2 - 3 } \cline { 5 - 6 } & $T_{f}$ & $2.30(0.0201)$ & & 114.27 & $1.20 \cdot 10^{-76}$ & $2.2621-2.3426$ \\
\hline \multirow{3}{*}{$20 \% \mathrm{hv}$} & $T_{c i}$ & $4.20(0.0978)$ & \multirow{4}{*}{0.99} & 42.91 & $2.06 \cdot 10^{-49}$ & $4.0026-4.39334$ \\
\cline { 2 - 3 } \cline { 5 - 6 } & $T_{c e}$ & $4.49(0.1012)$ & 44.40 & $2.40 \cdot 10^{-50}$ & $4.2918-4.6961$ \\
\cline { 2 - 3 } & $T_{f}$ & $2.40(0.0310)$ & & 77.32 & $1.06 \cdot 10^{-65}$ & $2.3357-2.4596$ \\
\hline \multirow{3}{*}{$100 \% \mathrm{pc}$} & $T_{c i}$ & $3.67(0.0466)$ & & 78.74 & $5.46 \cdot 10^{-67}$ & $3.5754-3.7614$ \\
\cline { 2 - 3 } & $T_{c e}$ & $3.94(0.0482)$ & 81.85 & $4.35 \cdot 10^{-68}$ & $3.8464-4.0387$ \\
\cline { 2 - 3 } & $T_{f}$ & $2.19(0.0143)$ & & 153.21 & $5.84 \cdot 10^{-86}$ & $2.1656-2.2228$ \\
\hline
\end{tabular}

Note: hv - stands for heavy vehicles; $\mathrm{pc}$ - stands for passenger cars; $\alpha$ - significance level. 
a)

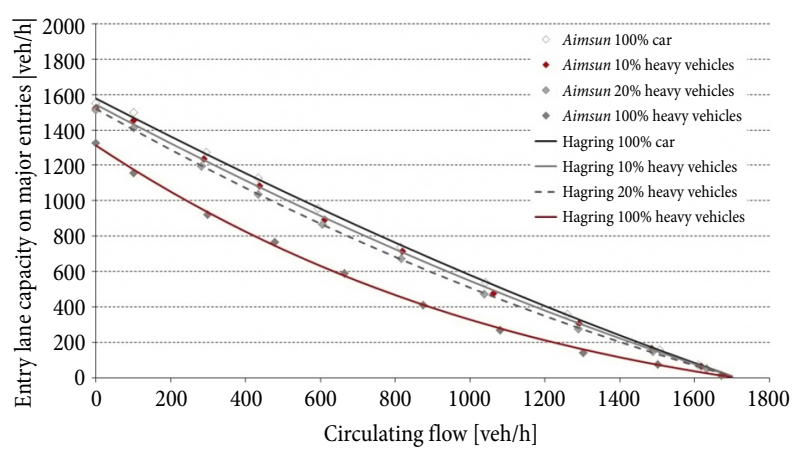

b)

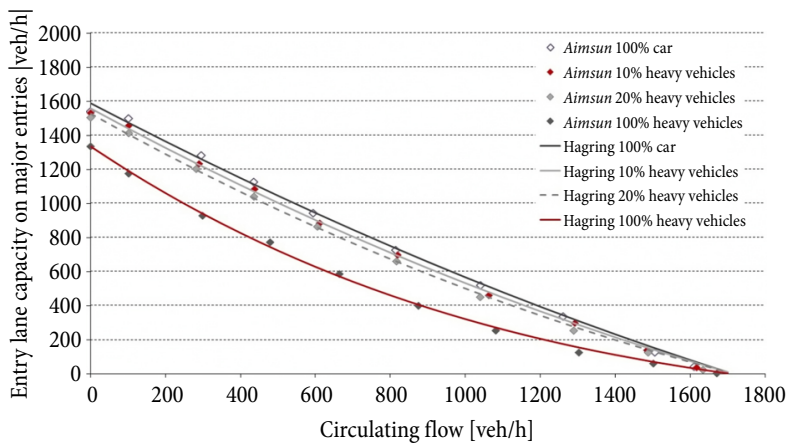

Fig. 5. Simulated points and capacity functions for the base and mix curves: a - the left-lane on major entries; $\mathrm{b}$ - the right-lane on major entries

\subsection{Modelling Results}

The PCEs for the subject type of heavy vehicle and for the explored mixed fleets were estimated from Eq. (3) for each entry lane at the at-grade turbo-roundabout. Fig. 6 shows the resulting PCEs for the left-lane on major entries (Fig. 6a) and for the right-lane on minor entries (Fig. 6b); in both cases $E_{t}$ is depending on the whole circulating flow $Q_{c}$.

For the left-lane on minor entries $E_{t}$ is depending on the inner $Q_{c i}$ and the outer circulating flow $Q_{c e}$ of the circulatory roadway; so $E_{t}$ are represented by surfaces (Fig. 7).

Results show that PCEs are sensitive to the variables here examined: in usual operational conditions ( $20 \%$ and $10 \%$ of heavy vehicles in the entry demand), $E_{t}$ resulted below 2 for each lane on major entries (Fig. 6a); higher values could be reached for an (unrealistic) traffic demand made of heavy vehicles only, or in saturated conditions for the circulating flow $\left(Q_{c e}=1700 \mathrm{veh} / \mathrm{h}\right)$. In turn, for the right-lane on minor entries, an $E_{t}$ of 4 is reached in usual operational conditions (Fig. 6b).

Assuming $E_{t}=2$, as Highway Capacity Manual (2010) suggests for roundabouts, the impact of heavy vehicles on the quality of traffic flow would be overestimated for left- and right-lane on major entries (Fig. 6a) and underestimated for the right-lane on minor entries (Fig. 6b). For the left-lane on minor entries (Fig. 7) and in usual operational conditions (namely mixed fleets with $10 \%$ and $20 \%$ of heavy vehicles), an $E_{t}$ of 4.5 is reached. Being constant the value of $Q_{c e}$, PCE grows a)

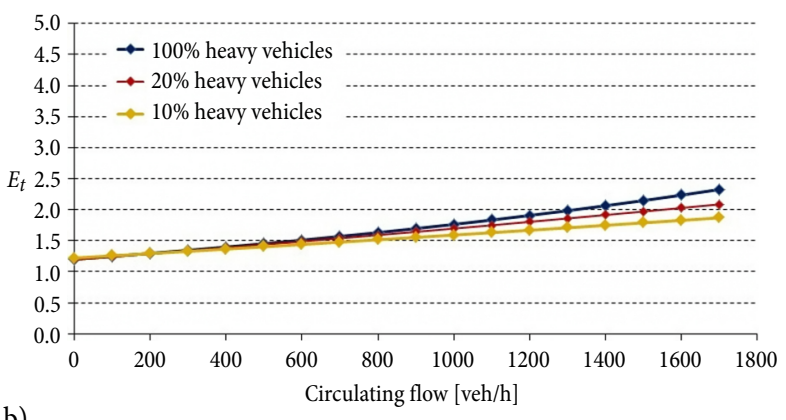

b)

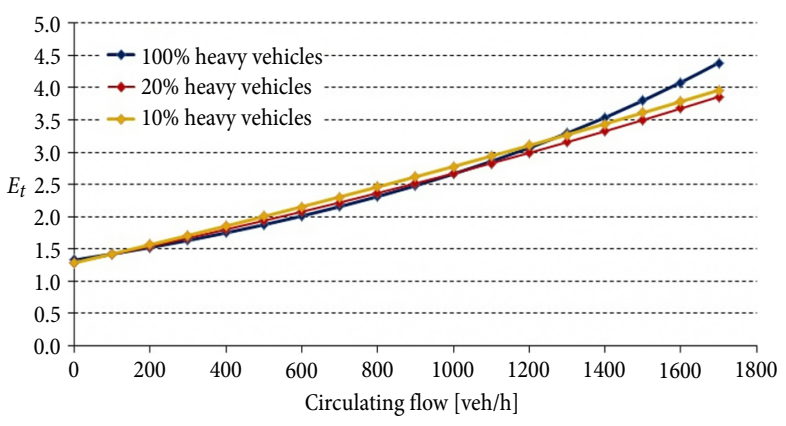

Fig. 6. PCE estimations: a - left-lane on major entries; $\mathrm{b}$ - right-lane on minor entries

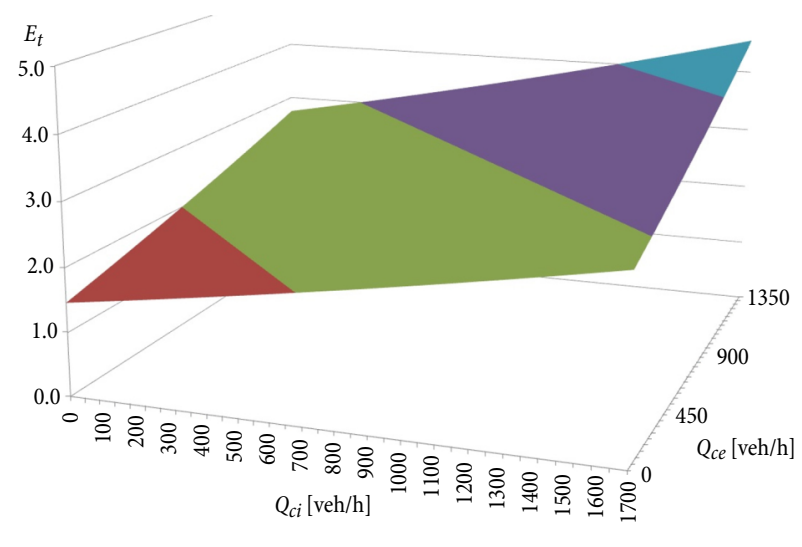

$4.00-5.00 \square 3.00-4.00 \quad \square .00-3.00 \square$
$\square$

Fig. 7. $E_{t}$ for the left lane on minor entry (case $-20 \%$ of heavy vehicles)

when $Q_{c i}$ grows; similar trend was observed for PCEs when $Q_{c e}$ increases (being constant the value of $Q_{c i}$ ). Thus, assuming an $E_{t}=2$, as Highway Capacity Manual (2010) suggests for roundabouts, a significant underestimation of the impact of heavy vehicles on the quality of traffic flow may happen.

\section{Conclusions}

Limited scientific literature and few studies related to the calculation of PCEs for heavy vehicles for roundabouts represent a gap in knowledge. This article addresses the question of how to estimate PCEs for heavy vehicles driving turbo-roundabouts. Starting from the initial belief that the constraints to the vehicular trajectories imposed by the turbo-roundabout design imply that the impact of heavy vehicles on the quality of traf- 
fic flow is higher if compared to other roundabouts, a microsimulation-based method was applied to evaluate the variation of the quality of traffic in presence of mixed fleets, each having different percentages of heavy vehicles. Based on the output of multiple runs of the different mixed fleet scenarios simulated in Aimsun, capacity functions for each lane from the turbo-roundabout entries were developed and the PCEs for heavy vehicles driving turbo-roundabouts were then calculated by comparing a fleet of passenger cars only with a mixed fleet characterised each time by different percentages of heavy vehicles. The capacity functions developed for each entry lane allowed us to determine how the PCE varies with the percentage of heavy vehicles and circulating flows. The investigation revealed interesting and to some extent unexpected implications for PCEs for heavy vehicles when estimated in the operational conditions that characterise the turbo-roundabout. When the traffic stream contains a significant number of heavy vehicles, a larger PCE effect was expected. Assuming the value of PCE for roundabouts provided by the $2010 \mathrm{HCM}\left(E_{t}=\right.$ 2 ), overestimation of the impact of heavy vehicles on the quality of traffic flow can happen for left- and right-lane on major entries and underestimation of the impact of heavy vehicles can happen for the right- and the left-lane on minor entries. Since assuming the values of PCEs for roundabouts provided by the $2010 \mathrm{HCM}$ the effect of heavy vehicles on the quality of the traffic flow could be misinterpreted; locally calibrated, country-specific, PCEs should be preferred for capacity calculations.

The reader should be advised that in this application, the Authors were more concerned with the explanatory power of the model than with the absolute accuracy of their estimates and, as such, a highly realistic representation of existing turbo-roundabouts was not deemed essential. According to Vasconcelos et al. (2012), the model was only required to be validated both at the level of conceptual validation, that is the model representation of the problem entity was reasonable, and at the level of computerized model verification, that is the computer programming and implementation of the conceptual model were correct. At last, it should be emphasized that an important aspect of the research consists in having identified a methodology for assessing the impact of heavy vehicles on the quality of traffic flow at turbo-roundabouts, that could be also applied to different type of intersections and roundabouts in order to improve the geometry design.

\section{References}

Adnan, M. 2014. Passenger car equivalent factors in heterogenous traffic environment-are we using the right numbers?, Procedia - Engineering 77: 106-113. http://doi.org/10.1016/j.proeng.2014.07.004

Al-Kaisy, A. 2006. Passenger car equivalents for heavy vehicles at freeways and multilane highways: some critical issues, ITE Journal 76(3): 40-43.

Al-Kaisy, A. F.; Hall, F. L.; Reisman, E. S. 2002. Developing passenger car equivalents for heavy vehicles on freeways during queue discharge flow, Transportation Research Part A: Policy and Practice 36(8): 725-742. http://doi.org/10.1016/S0965-8564(01)00032-5
Al-Kaisy, A.; Jung, Y.; Rakha, H. 2005. Developing passenger car equivalency factors for heavy vehicles during congestion, Journal of Transportation Engineering 131(7): 514-523. http://doi.org/10.1061/(ASCE)0733-947X(2005)131:7(514)

Anwaar, A.; Van Boxel, D.; Volovski, M.; Anastasopoulos, P. C.; Labi, S.; Sinha, K. C. 2011. Using lagging headways to estimate passenger car equivalents on basic freeway sections, Journal of Transportation of the Institute of Transportation Engineers 2(1): 1-17.

Barceló, J. 2010. Fundamentals of Traffic Simulation. Springer. $442 \mathrm{p}$.

Bastos Silva, A.; Mariano, P.; Silva, J. P. 2015. Performance assessment of turbo-roundabouts in corridors, Transportation Research Procedia 10: 124-133. http://doi.org/10.1016/j.trpro.2015.09.062

Craus, J.; Polus, A.; Grinberg, I. 1980. A revised method for the determination of passenger car equivalencies, Transportation Research Part A: General 14(4): 241-246. http://doi.org/10.1016/0191-2607(80)90040-0

CROW. 2008. Turborotondes. Artikelnummer 257. CROW Kennisplatform. Nederland. 128 p. (in Dutch).

Cunagin, W. D.; Messer, C. J. 1983. Passenger-car equivalents for rural highways, Transportation Research Record: Journal of the Transportation Research Board 905: 61-68.

Demarchi, S.; Setti, J. 2003. Limitations of passenger-car equivalent derivation for traffic streams with more than one truck type, Transportation Research Record: Journal of the Transportation Research Board 1852: 96-104. http://doi.org/10.3141/1852-13

Elefteriadou, L.; Torbic, D.; Webster, N. 1997. Development of passenger car equivalents for freeways, two-lane highways, and arterials, Transportation Research Record: Journal of the Transportation Research Board 1572: 51-58.

http://doi.org/10.3141/1572-07

Fortuijn, L. 2009. Turbo roundabouts: estimation of capacity, Transportation Research Record: Journal of the Transportation Research Board 2130: 83-92. http://doi.org/10.3141/2130-11

Geistefeldt, J. 2009. Estimation of passenger car equivalents based on capacity variability, Transportation Research Record: Journal of the Transportation Research Board 2130: 1-6. http://doi.org/10.3141/2130-01

Gingrich, M. A.; Dion, F. 2012. Predicting multimodal roundabout operations by combining deterministic and microsimulation models, Journal of Transportation of the Institute of Transportation Engineers 3(1): 1-14.

Giuffrè, O.; Granà, A; Marino, S. 2012. Turbo-roundabouts vs roundabouts performance level, Procedia - Social and Behavioral Sciences 53: 590-600. http://doi.org/10.1016/j.sbspro.2012.09.909

Hagring, O. 1998. A further generalization of Tanner's formula, Transportation Research Part B: Methodological 32(6): 423 429. http://doi.org/10.1016/S0191-2615(98)00010-1

Highway Capacity Manual. 2010. Transportation Research Board. 5th edition. 1650 p.

Highway Capacity Manual. 2000. Transportation Research Board. $1134 \mathrm{p}$.

Highway Capacity Manual: Practical Applications of Research. 1950. Highway Research Board, Washington, DC.

Highway Capacity Manual: Special Report 209. 1994. Transportation Research Board, Washington, DC.

Highway Capacity Manual: Special Report 87. 1965. Highway Research Board, Washington, DC. 
Huber, M. J. 1982. Estimation of passenger-car equivalents of trucks in traffic stream, Transportation Research Record: Journal of the Transportation Research Board 869: 60-70.

Jia, F.-Y.; Yan, L.; Dong, J.-S.; Guan, Z.-C. 2008. Analyze research on signal control based on vehicular emission of urban road roundabout, Acta Scientiarum Natralium Universitatis Sunyatseni 47(2): 161-164.

Keller, E. L.; Saklas, J. G. 1984. Passenger car equivalents from network simulation, Journal of Transportation Engineering 110(4): 397-411.

http://doi.org/10.1061/(ASCE)0733-947X(1984)110:4(397)

Lee, C. 2014. Passenger car equivalents for heavy vehicles at roundabouts: estimation and application to capacity prediction, in TRB 93rd Annual Meeting Compendium of $\mathrm{Pa}$ pers, 12-16 January 2014, Washington, DC, 1-16.

Macioszek, E. 2012. Geometrical determinants of car equivalents for heavy vehicles crossing circular intersections, in J. Mikulski (Ed.). Telematics in the Transport Environment: 12th International Conference on Transport Systems Telematics, TST 2012, Selected Papers, 10-13 October 2012, Katowice-Ustroń, Poland, 221-228. http://doi.org/10.1007/978-3-642-34050-5_25

Mauro, R.; Cattani, M.; Guerrieri, M. 2015. Evaluation of the safety performance of turbo roundabouts by means of a potential accident rate model, The Baltic Journal of Road and Bridge Engineering 10(1): 28-38. http://doi.org/10.3846/bjrbe.2015.04

Mauro, R.; Giuffrè, O.; Granà, A.; Chiappone, S. 2014. A statistical approach for calibrating a microsimulation model for freeways, WSEAS Transactions on Environment and Development 10: 496-508.

Praveen, P. S.; Arasan, V. T. 2013. Influence of traffic mix on PCU value of vehicles under heterogeneous traffic conditions, International Journal for Traffic and Transport Engineering 3(3): 302-330. http://doi.org/10.7708/ijtte.2013.3(3).07

Tollazzi, T. 2015. Alternative Types of Roundabouts: an Informational Guide. Springer International Publishing. 206 p. http://doi.org/10.1007/978-3-319-09084-9

Vasconcelos, A. L. P.; Bastos Silva, A.; Seco, Á. J. M. 2014. Capacity of normal and turbo-roundabouts: comparative analysis, Proceedings of the Institution of Civil Engineers Transport 167(2): 88-99. http://doi.org/10.1680/tran.12.00003

Vasconcelos, A. L. P.; Seco, Á. J. M.; Bastos Silva, A. 2013. Comparison of procedures to estimate critical headways at roundabouts, Promet - Traffic\&Transportation 25(1): 43-53. http://doi.org/10.7307/ptt.v25i1.1246

Vasconcelos, L.; Seco; Á.; Bastos Silva, A.; Abreu, T.; Silva, J. P. 2012. A comparison of roundabout capacity models, in The 23rd IASTED International Conference on Modelling and Simulation (MS 2012), 3-5 July 2012, Banff, Canada, 165-170. http://doi.org/10.2316/p.2012.783-023

Webster, N.; Elefteriadou, L. 1999. A simulation study of truck passenger car equivalents (PCE) on basic freeway sections, Transportation Research Part B: Methodological 33(5): 323336. http://doi.org/10.1016/S0965-8564(98)00036-6

Zeņina, N.; Merkuryev, Y. 2009. Analysis of simulation input and output to compare simulation tools, Mechanics, Transport, Communications: Academic Journal (3): V-4-V-9.

Zhang, K.; Excell, A. 2013. Modelling a complex give-way situation - AIMSUN vs LINSIG, Road \& Transport Research: $a$ Journal of Australian and New Zealand Research and Practice 22(2): 16-26. 